\title{
Research on the Wireless Sensor Network System Based on Internet of Things
}

\author{
Di Huang $^{a}$, Jiang Yongxing ${ }^{b}$ and Wang Ziyue ${ }^{c}$ \\ International School, Beijing University of Posts and Telecommunications, Beijing 102209, China \\ ahuangdi@bupt.edu.cn, b1512632413@qq.com, ${ }^{c} 2012212883 @$ bupt.edu.cn
}

Keywords: Internet of Things, wireless sensor network, sensor node.

\begin{abstract}
As the third information industry after the computer and Internet revolution, the Internet of Things is coming which brings us into a new era in which everything can be identified and connected and things can exchange information and make decisions by themselves. As a core technology of the Internet of Things, the research of wireless sensor network becomes a top priority, especially for the security of wireless sensor network. In this paper, the structural characteristics of wireless sensor network and the hardware structure design of sensor node are analyzed, and then based on the Internet of Things, we discuss the application structure of wireless sensor network system.
\end{abstract}

\section{Introduction}

With the rapid development of Internet industry and the sharp increase in Internet access devices, meeting the needs of the business system to a large extent and operating system access to the underlying Internet equipment is quite difficult. Due to the lack of access platforms, applications need to implement a wide range of devices to access agreements in the development process to meet the access to different devices. This dilemma is a serious impediment to the further development of the Internet industry. A large number of sensors can be networked in many applications that require unattended operations, hence producing a wireless sensor network (WSN)[1]. With the development of wireless communication, sensor technology, embedded applications and microelectronics technology, WSN can gain information at any time, any place, any environmental conditions required, and lay the foundation for the development of Internet of Things (IoT). And WSN has a lot of strong technical advantages, such as self-organization, quick deployment, high fault-tolerant and concealment, so lend itself well to battlefield target positioning and physiological data collection, intelligent transportation systems and ocean exploration, and many other fields [2-3].

This paper does research on the wireless sensor network technology, including the architecture of wireless sensor network system, hardware structure design of sensor node, and the application structure of WSNs based on Internet of Things. In the final section, we conclude this paper.

\section{Wireless sensor network system technology}

Wireless sensor network system is an important part of Internet of things aimed at perceiving, this perception is mainly through the nodes deployed in various ways in the individual perceived objects inside or nearby to get various information which exists in the physical world [4]. Typically, WSNs contain hundreds or thousands of sensor nodes, and these sensors have the ability to communicate either among each other or directly to an external base station. Deploying these sensor nodes to constitute networks through the way of self-organization, integrating sensor with different function, data processing unit and communications unit, and deploying among the nodes. Basically, each sensor node comprises sensing, processing, transmission, mobilizer, position finding system, and power units. A greater number of sensors allows for sensing over larger geographical regions with greater accuracy. Sensor nodes coordinate among themselves to produce high-quality information about the physical environment. The category of perception main include temperature, intensity of light, humidity, electromagnetic waves and acoustic wave. These data are to obtain measurements of environmental parameters [5]. 
WSNs network architecture is shown in Figure.1. Huge number of sensor nodes randomly spread or deployed in the monitoring area of the hand through the way of self-organization network [6]. Detected by a sensor node data is within the area of network nodes in multi-hop routing transmission finally reach the sink, there may be multiple nodes during the transfer process to perform fusion and compression, and finally via satellite, Internet or wireless access server Terminal Management node. Users can manage WSNs node configured by management, task dissemination and feedback operations such as security controls.

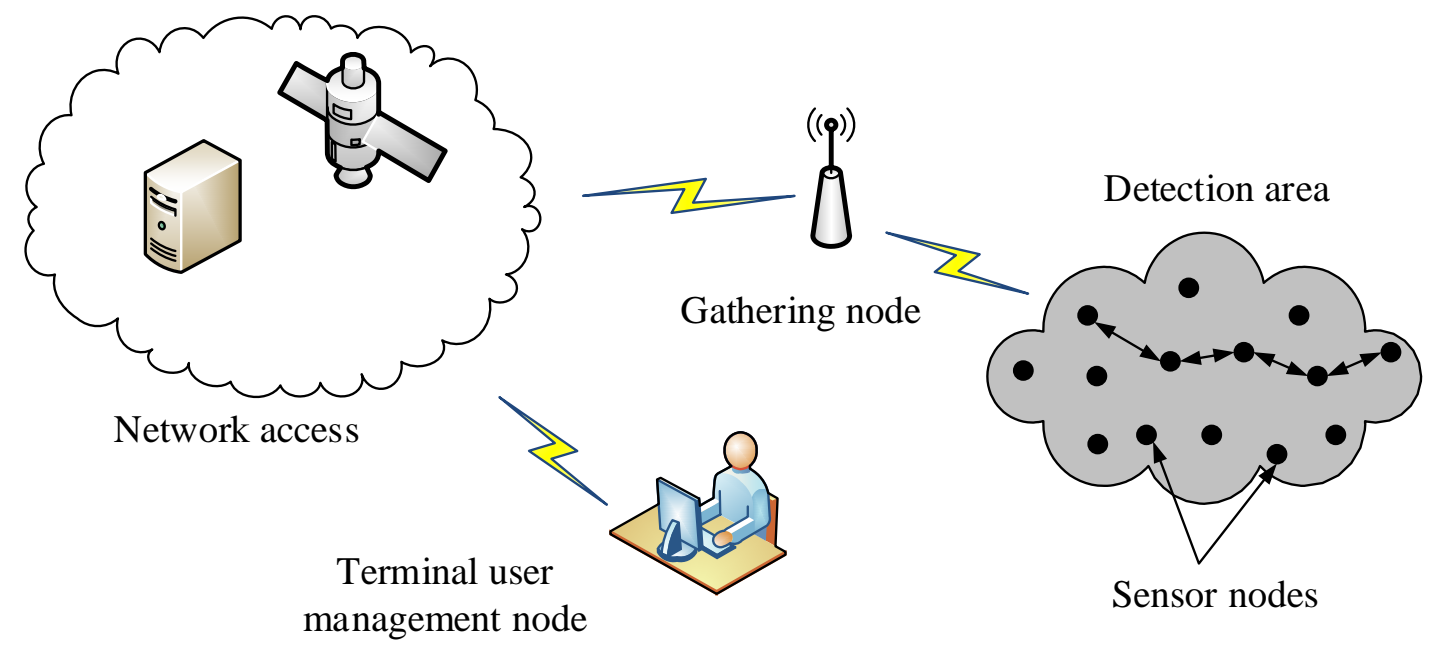

Fig. 1 Architecture of wireless sensor network system

In this paper, we consider a wireless sensor network with a single mobile sink. We assume (1) sensor nodes are deployed on a flat surface, and their position can be determined by locating techniques; (2) the communication range of sensor nodes is the same as that of the communication radius; (3) communication is the communication radius of stable and mobile sink and all sensor nodes are the same; (4) in the data collection period, each sensor node generates the same amount of data, and the mobile receiver will take the same time to pick them up.

\section{Hardware structure design of sensor node}

Wireless sensor networks have different design goals with the traditional wireless networks (such as WLAN and cellular mobile phone networks). The traditional wireless network design objective is to maximize the bandwidth utilization by optimizing the routing and resource management strategies, and also to provide a certain degree of service quality assurance for the user in a highly mobile environment. In wireless sensor networks, in addition to a small amount of nodes need to go beyond, most nodes are static. Because they are usually run by people not close to bad or even dangerous to the remote environment, cannot replace energy, design effective strategies to extend the network life cycle to become the core issue of wireless sensor networks, which is the design of the problem. Wireless sensor networks, which is different from the traditional network of technical requirements, sensor networks in the data center, the traditional network to transmit data purposes. For this, we made the hardware structure design of sensor node, which is shown in Figure.2. 


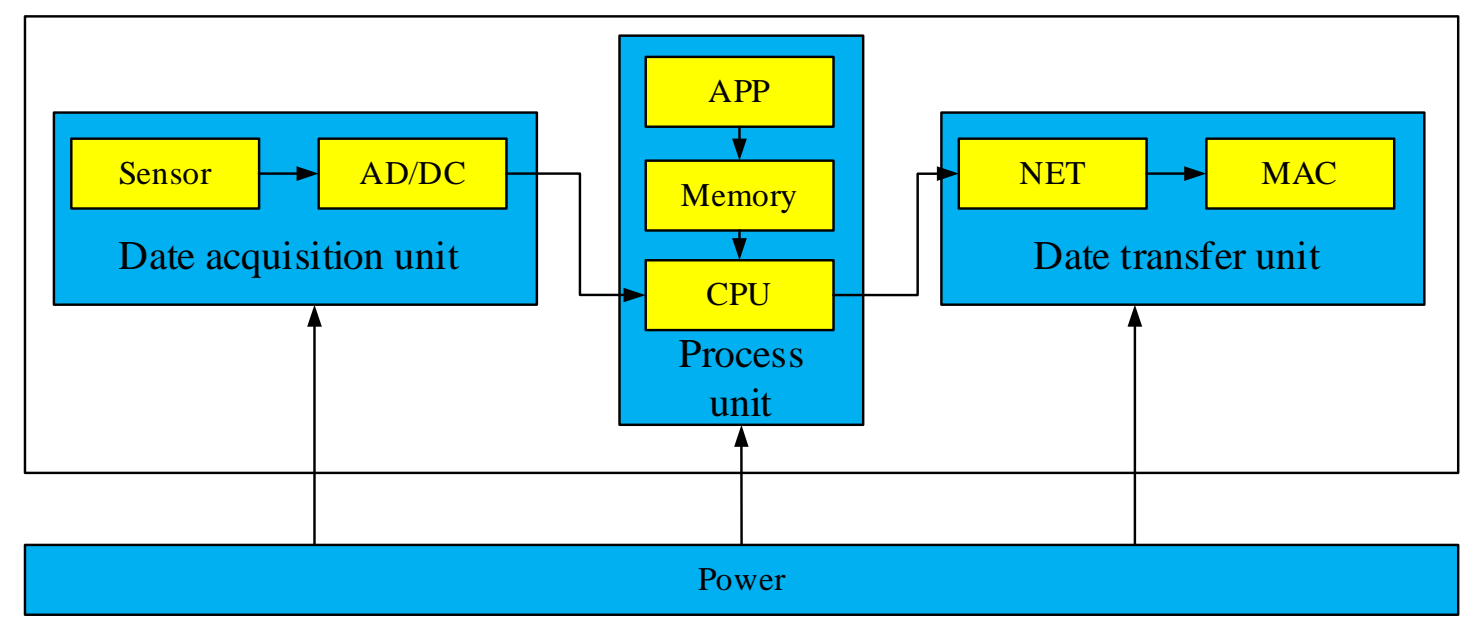

Fig. 2 Hardware structure design of sensor node

In different applications, sensor nodes are different, but are usually composed of data sets. Power of data processing, data transmission, and four parts (as shown in Figure). By monitoring the physical form of sensor type, signal processor is usually selected embedded CPU or micro controller, data transmission unit based low power, short distance wireless communication module, sensor node hardware structure design for various industries in the design of sensor nodes, we chose the design of the node has five main modules: processor, radio frequency communications, power management, expand I /O and auxiliary storage.

\section{The Application structure of wireless sensor network system based on Internet of Things}

In dense sensor networks, the distance between adjacent nodes is very short, and low power consumption of multi hop communication mode to save power consumption, while the concealment of communication, but also in order to avoid long-distance wireless communication is vulnerable to external noise interference. These unique requirements and constraints of sensor network architecture are proposed for the design of new requirements. The application of wireless sensor network system based on the Internet of Things, in the bottom of the sensor nodes, it performs a number of common data acquisition, computing and networking capabilities and the specific application of the sense of perception. The sensor nodes are densely arranged in the region of interest, and these cells are too far apart. Sensor network application architecture is shown in Figure.3.

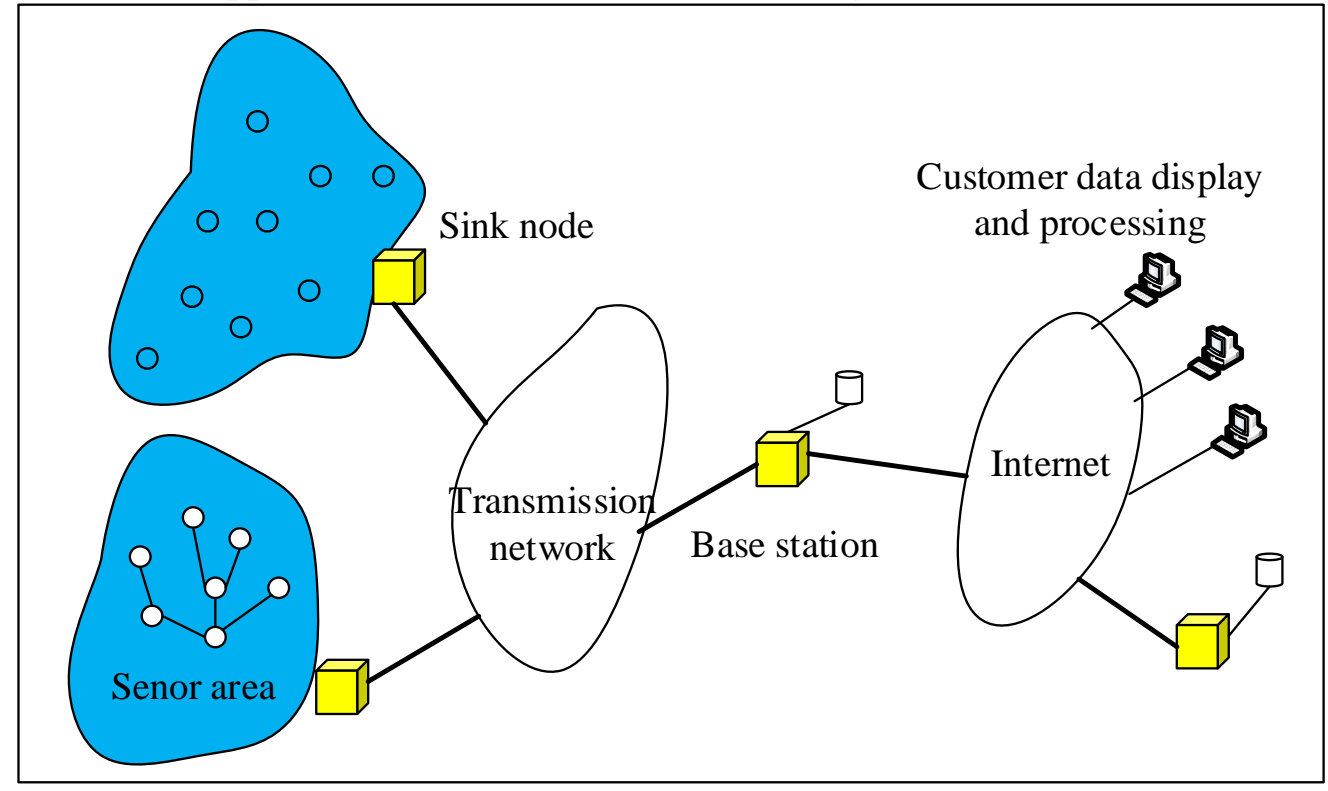

Fig. 3 Application structure of wireless sensor network system based on Internet of Things 
In order to adapt to a wide range of applications, the focus of the traditional network design will be all functions, the terminal system on the network related processing, intermediate nodes are responsible for forwarding data packets, but it is not a reasonable choice of sensor network.

\section{Conclusion}

With the rapid development of science and technology development, WSNs have become ubiquitous and are being used in a broad array of application domains, including healthcare, agriculture, surveillance, and security. In this paper, we first analyze the structural characteristics of wireless sensor network and the hardware structure design of sensor node, and then based on Internet of Things, the application structure of WSNs and some new ideas for future development of WSNs were put forward. Finally, we do hope that this research will be helpful for researchers in the field, helping them to understand the huge potential of WSNs and IoT, and devising innovative technical solutions able to turn IoT from a research vision into reality.

\section{References}

[1] Hong S, Kim D, Ha M, et al. SNAIL: an IP-based wireless sensor network approach to the internet of things[J]. Wireless Communications, IEEE, 2010, 17(6): 34-42.

[2] Atzori L, Iera A, Morabito G. The internet of things: A survey[J]. Computer networks, 2010, 54(15): 2787-2805.

[3] Li L, Hu Z Y. The Research of Wireless Sensor Network System Based on Internet of Things[C]//Applied Mechanics and Materials. 2014, 602: 3813-3816.

[4] Mainetti L, Patrono L, Vilei A. Evolution of wireless sensor networks towards the internet of things: A survey[C]//Software, Telecommunications and Computer Networks (SoftCOM), 2011 19th International Conference on. IEEE, 2011: 1-6.

[5] Li S, Da Xu L, Wang X. Compressed sensing signal and data acquisition in wireless sensor networks and internet of things[J]. Industrial Informatics, IEEE Transactions on, 2013, 9(4): 2177-2186.

[6] Gubbi J, Buyya R, Marusic S, et al. Internet of Things (IoT): A vision, architectural elements, and future directions[J]. Future Generation Computer Systems, 2013, 29(7): 1645-1660. 